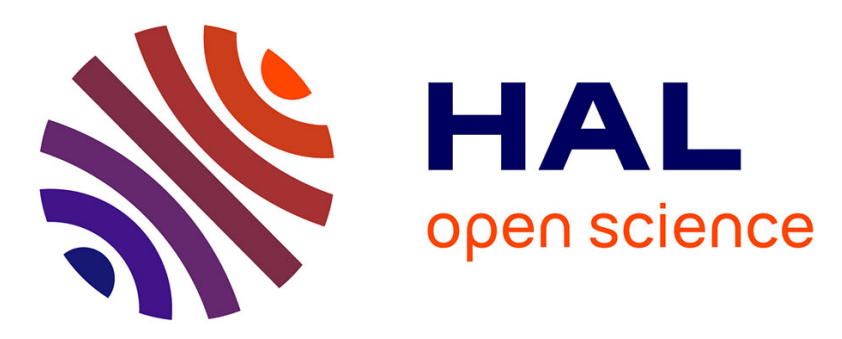

\title{
3D Haptic Rendering of Tissues for Epidural Needle Insertion using an Electro-Pneumatic 7 Degrees of Freedom Device
}

Pierre-Jean Alès Roux, Nicolas Herzig, Arnaud Lelevé, Richard Moreau, Christian Bauer

\section{To cite this version:}

Pierre-Jean Alès Roux, Nicolas Herzig, Arnaud Lelevé, Richard Moreau, Christian Bauer. 3D Haptic Rendering of Tissues for Epidural Needle Insertion using an Electro-Pneumatic 7 Degrees of Freedom Device. 2016 IEEE IROS, Oct 2016, Daejeon, South Korea. 10.1109/IROS.2016.7759760 . hal01340723

\section{HAL Id: hal-01340723 \\ https://hal.science/hal-01340723}

Submitted on 7 Jan 2019

HAL is a multi-disciplinary open access archive for the deposit and dissemination of scientific research documents, whether they are published or not. The documents may come from teaching and research institutions in France or abroad, or from public or private research centers.
L'archive ouverte pluridisciplinaire HAL, est destinée au dépôt et à la diffusion de documents scientifiques de niveau recherche, publiés ou non, émanant des établissements d'enseignement et de recherche français ou étrangers, des laboratoires publics ou privés. 


\title{
3D Haptic Rendering of Tissues for Epidural Needle Insertion using an Electro-Pneumatic 7 Degrees of Freedom Device
}

\author{
Pierre-Jean Alès ${ }^{1}$, Nicolas Herzig ${ }^{2}$, Arnaud Lelevé ${ }^{2}$, Richard Moreau ${ }^{2}$ and Christian Bauer ${ }^{3}$
}

\begin{abstract}
Epidural anaesthesia is a medical gesture commonly performed by an anaesthesiologist. However, it remains one of the most difficult gestures to master for medical students. Given the lack of sufficiently realistic training devices available for future physicians, we propose a new haptic simulator which reproduces the haptic sensations felt by anaesthesiologists when performing this kind of operation. The originality of this simulator is the coupling of a Geomagic Touch $^{\circledR}$ device with a pneumatic cylinder to reproduce the "Loss of Resistance" phenomenon which helps the physician to control the needle depth. In this paper, we introduce the parametric 3D model of the region of interest and the control laws used jointly. Even though this device could not reproduce the right level of forces required in this type of anaesthesia, an anaesthesiologist involved in the project gave positive feedback about its haptic tissue rendering.
\end{abstract}

\section{INTRODUCTION}

The epidural anaesthesia is a loco-regional anaesthesia commonly used as a pain relief for women in labour and as a mean to provide anaesthesia in different surgeries such as the hip, knee or rib fracture surgeries, or amputations. It can be performed at different locations along the spine depending on the application. For instance, approximately 280000 epidural anaesthesia (see Fig. 1) are performed yearly in the National Health Services in England [1], [2].

As described by Manoharan and Tran in [2] and [3], the epidural anesthesia is a two-stage procedure. First, the practitioner inserts the needle through the skin, the fat, the supraspinous ligament, the intraspinous ligament, the ligamentum flavum until it finally reaches the epidural space. In order to recognize the latter, the physician uses the "Loss Of Resistance" technique. This technique consists in injecting air or physiological liquid through the needle by way of a syringe. This fluid sparsely leaks while the needle is crossing the preliminary layers. Once the needle has reached the epidural space, the fluid disseminates and the physician feels it instantaneously as the syringe empties quickly. He must stop inserting the needle at this right moment. The second stage consists in removing the syringe, inserting a catheter through the needle and then removing the needle. The anesthetic will be injected in the epidural space through this catheter.

\footnotetext{
${ }^{1} \mathrm{PhD}$ student, Department of Computer Science, University of Verona , Italy. Strada Le grazie 15, $37 \quad 134$ VERONA, Italy pierrejean.ales@univr.it

${ }^{2}$ Univ Lyon, INSA Lyon, Laboratoire Ampre (UMR 5005), F 69621, LYON, France \{nicolas.herzig, arnaud.leleve, richard.moreau\}einsa-lyon.fr

${ }^{3}$ Department of Anesthesia and Intensive Care, Hôpital de la Croix-Rousse, Hospices civils de Lyon, LYON, France christian.bauerechu-lyon. fr
}

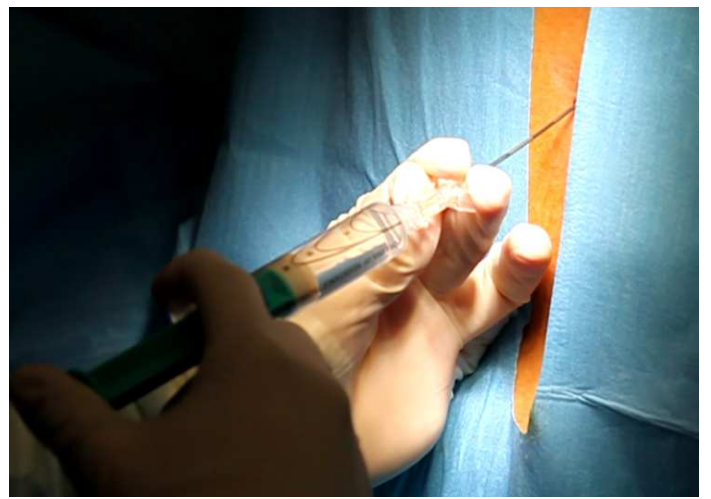

Fig. 1: Epidural anesthesia insertion

In the event the practitioner continues to push after having reached the epidural space, the needle will enter the dura and injure the patient. The most common complication is the Post-Dural Puncture Headache that can last up to three weeks. It occurs in $1 \%$ of the cases in the UK with a wide variation depending on the concerned medical unit. The patient can expect full recovery in two weeks to three months. "The epidural anesthesia is one of the most difficult skill to acquire by medical students with a success rate of barely $80 \%$ after 90 attempts" [1], [4], [5].

Medical students usually learn this gesture by way of cadavers, animals or passive simulators. Unfortunately, cadavers and animals are not always available and do not reproduce accurately the haptic response of a living human body. To get a precise view of the current state of the art about modern epidural simulation, we performed a survey over 35 simulators for the epidural insertion or similar procedures, which can be sorted, according to [1], into two categories: passive and active simulators.

The passive simulators are available at a relatively low cost and provide a realistic representation of the human anatomy allowing the user to palpate the patient and to choose the needle point of insertion. The biological layers are represented using materials with different properties. In addition, they can sometimes be used for several medical interventions such as the epidural anaesthesia, lumbar puncture and caudal anaesthesia. However, these simulators present important drawbacks: the reaction forces felt by the trainer when inserting the needle are not accurate and it is necessary to change the material after several trials, which is costly in the long run.

The active simulators use haptic interfaces, calculators and actuators to provide a better feeling to the user. In that case, 


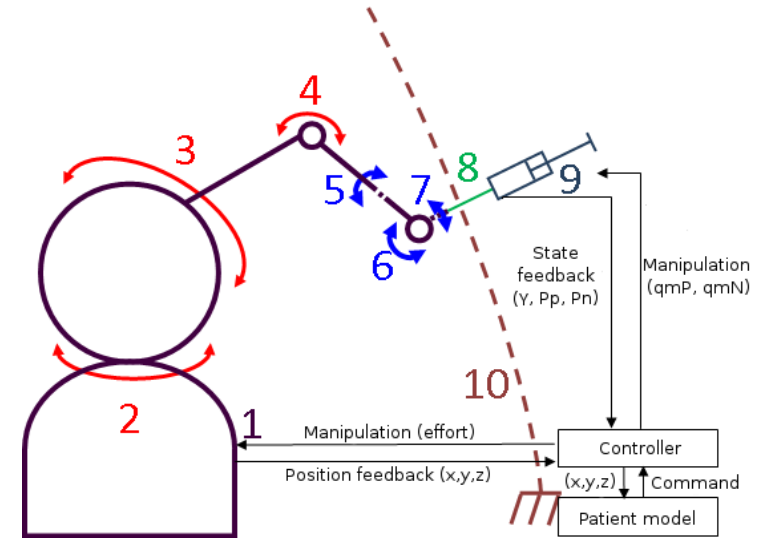

Fig. 2: General scheme of the prototype

\begin{tabular}{|l|l|}
\hline Element & Definition \\
\hline 1 & Electric interface \\
\hline $2 ; 3 ; 4$ & Motorized pivot joints \\
\hline $5 ; 6 ; 7$ & Passive pivot joints \\
\hline 8 & Needle \\
\hline 9 & Pneumatic actuator (syringe) \\
\hline 10 & Back of the patient \\
\hline$x, y, z$ & Position of the tip of the needle [m] \\
\hline$Y$ & Rod position of the pneumatic actuator $(\mathrm{PA})[\mathrm{m}]$ \\
\hline$q_{m}, q_{m}$ & $\begin{array}{l}\text { Air mass flow rates entering into each } \\
\text { chamber of the PA [kg/s] }\end{array}$ \\
\hline$P_{p}, P_{n}$ & Pressure in each of the chambers of the PA [Pa] \\
\hline
\end{tabular}

TABLE I: Legend of figure 2

the patient's anatomy is generally not precisely physically represented, sometimes only the external appearance. For mechanical reasons, the user cannot choose the insertion point nor palpate the patient. Examples of such simulators are provided in [6], [7] and [8]. The main disadvantage of these simulators is their acquisition cost compared to passive ones. To the best of the authors' knowledge, the existing simulators replicate the forces applied to the needle and the behavior of the syringe is approximated in a binary way: either the liquid flows out or not. However, the stiffness felt on the syringe in the different layers is used by the practitioner to locate the epidural space.

In this paper we study the feasibility of coupling an electric interface with a pneumatic actuator in order to display the forces applied on the needle and the stiffness variations of the syringe's piston according to a parametric 3D model of the back of the patient. This be will be useful to obtain a realistic simulator to help medical students to safely train themselves on the epidural insertion procedure. A schematic representation of a prototype featuring a Geomagic Touch $^{\circledR}$ is provided in figure 2 and table I. Usually, the syringe is filled with air or a saline solution. In our work, we chose to work with air. Next section describes this parametric model and its parameters and section III details the control laws used with this model.

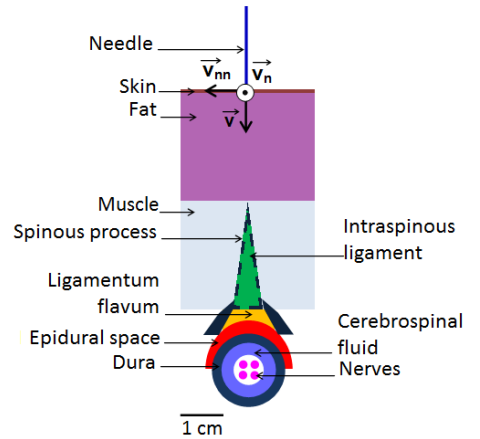

Fig. 3: Simplified model of a transverse cut in the lumbar region

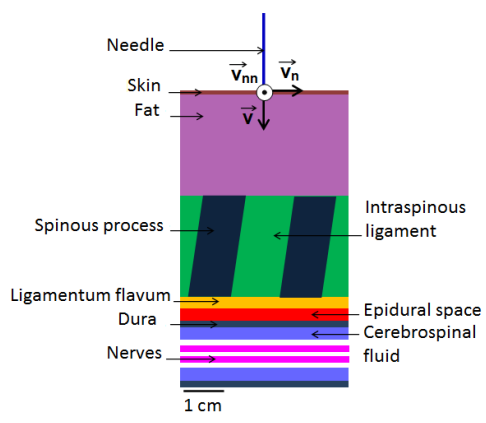

Fig. 4: Simplified model of a sagittal cut in the lumbar region

\section{ANATOMIC 3D MODEL}

\section{A. Expected performances}

Working in close collaboration with an anesthesiologist, we defined the expected performances of the device. First of all, it should reproduce the cutting and friction forces felt by the physician when the needle enters the patient's back. These efforts are exerted in the opposite direction of the axial movement of the needle. Secondly, it is important to limit the radial motion of the needle while giving the user the possibility to adjust its position. Thirdly, a good rendering of the contact with the vertebrae is required. Finally, the pneumatic actuator should reproduce the stiffness of the syringe and the liquid leakage in the patient's back.

In our work, we chose to uncouple the control laws: a first control law computes the radial forces while the second one computes the axial ones. These forces are summed up before being sent to the electric device actuators. The control law of the pneumatic actuator is independent from the control laws of the electric part. It only uses the position of the needle's tip to compute the command.

\section{B. Parametric force model}

A simplified parametric 3D model of the lumbar region of the patient has been established (see figures 3 and 4 ). A local frame coordinate $\left(\vec{v}, \vec{v}_{n}, \vec{v}_{n n}\right)$ has been chosen. Given the results already presented in the literature and the discussions with the anesthesiologist, a first parametric system has been established. It enables to adapt the modeled anatomy for 


\begin{tabular}{|c|c|}
\hline Parameters & Definition \\
\hline$z_{i}[\mathrm{~N} / \mathrm{m}]$ & Depth at which the needle enters the layer $i$ \\
\hline$B_{i}[\mathrm{Ns} / \mathrm{m}]$ & Viscosity in the layer $i$ \\
\hline$F_{a_{i}}[\mathrm{~N}]$ & Maximal force to be reached in layer $i$ \\
\hline \begin{tabular}{l|l}
$z_{m_{i}}$ & {$[\mathrm{~m}]$}
\end{tabular} & $\begin{array}{l}\text { Distance to cover in order to reach } F_{a_{i}} \\
\text { after a stop or a change of layer }\end{array}$ \\
\hline
\end{tabular}

TABLE II: Parameters used in the model to determine the cutting and friction forces

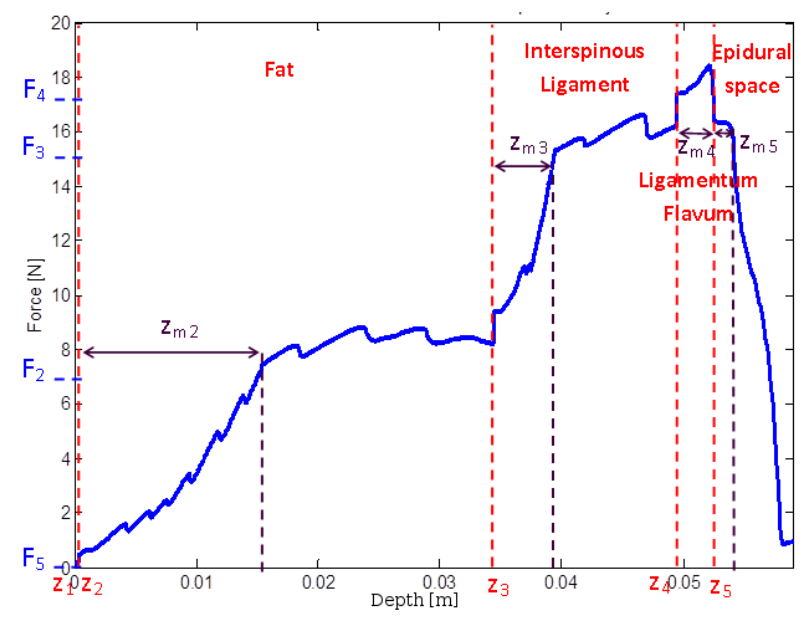

Fig. 5: Axial force profile generated during an epidural operation

different areas along the spine and for different patients (young/older, thin/fat, ...).

Some measurements performed by Brett in [7] and by Tran in [3] provide a reference for the axial force exerted on the needle by the physician. We used them to calibrate our system. This force ranges from 0 to $10 \mathrm{~N}$ or from 0 to $20 \mathrm{~N}$ depending on the type of patient. It is the sum of the cutting force, friction force and the stiffness reaction of the tissues. After some discussions with an anesthesiologist, it has been decided that the stiffness of the tissues will be neglected. Hence, four parameters are defined in each biological layer, visible in table II.

The force profile generated from this model is depicted in Fig. 5. It has been obtained by a trial performed by an anesthesiologist on a one degree of freedom device. During his gesture, the practitioner moved forward and stopped every few millimeters. This explains why the obtained force profile does not seem to be derivable. In addition, each trajectory between $z_{i}$ and $z_{m_{i}}$ is generated from a parabolic model instead of a linear one as the haptic rendering was found better from a physiological point of view (this provides the smooth aspect of the curve between each stop and layer crossing). In this case, the contact with the vertebrae is qualified as semi-hard. When the needles enters in contact with the vertebrae, it slowly sinks into it before getting stuck. In addition, it has been established that the needle cannot slip onto the vertebrae.

The limitation of the radial displacement is illustrated in Fig. 6. Using the frame coordinate defined in Fig. 3, it has

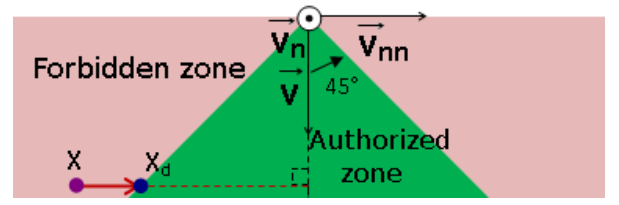

Fig. 6: Authorized and forbidden zones in the up-down direction

been established that the rotation around $\vec{v}_{n}$ and $\vec{v}_{n n}$ should be less than $45^{\circ}$ and $20^{\circ}$ respectively. In case the tip of the tool goes over this limit, the system guides it back into the authorized region. In this region, only a viscous reaction should be applied to the tool.

\section{Parametric model for the position and stiffness of the pneumatic actuator}

In this paragraph, we present the model used to compute the command to be applied on the pneumatic actuator. The stiffness of the pneumatic actuator in the different biological layers has been chosen for four different patients with the help of an anaesthesiologist: patient $\mathrm{A}$ is an obese person, patient $\mathrm{B}$ is an average person with hardly distinguishable tissues, patient $C$ is an average person with easily distinguishable tissues and patient $\mathrm{D}$ is an old person with calcification. The stiffness value for each layer for those patients is given in table III. These values were used as orders of magnitude to estimate the feasibility of a device and need to be refined with measurements in the future.

\begin{tabular}{|c|c|c|c|c|}
\hline Layer $\quad$ Patient & A & B & C & D \\
\hline Fat & 500 & 1450 & 1750 & 1147 \\
\hline Intraspinous ligament & 826.5 & 2170 & 2490 & 2452 \\
\hline Ligamentum flavum & 890 & 2216 & 2785 & 3511 \\
\hline Epidural space & 100 & 100 & 100 & 100 \\
\hline
\end{tabular}

TABLE III: Estimation of the stiffness felt on the syringe in the different biological layers $[\mathrm{N} / \mathrm{m}]$

Concerning the liquid flowing out of the syringe into each layer, a desired leak flow $q_{l}[\mathrm{~kg} / \mathrm{s}]$ is defined. According to the position of the tip of the needle in each layer, a different leak behavior requires to be reproduced. Hence when crossing the epidural space where the fluid can easily escape from the syringe, $q_{l}=q_{l \Psi}$ where $q_{l \Psi}$ is defined in eq. (1). The positive constant parameter $\Psi[\mathrm{kg} / \mathrm{s} / \mathrm{N}]$ proportionally links the leak flow to the force $F$ applied by the trainee on the syringe piston.

$$
q_{l \Psi}=\Psi \times F
$$

When crossing the preliminary layers, the liquid flows out of the syringe and diffuses into the patient tissues with a flow level depending on each layer and proportionnal to the force applied by the trainer on the syringe piston.

Next part describes the control laws used to implement the vertebrae contact, the radial movement limitation and the loss of resistance on the pneumatic actuator. 


\section{CONTROL LAWS}

\section{A. Radial forces}

The simplest way to implement the guidance feature is to use a proportional control law: $F_{p}=k\left(X_{d}-X\right)$, where $F_{p}$ is the force applied to the tool, $k[\mathrm{~N} / \mathrm{m}]$ mimics a stiffness, $X_{d}$ is the desired position of the tool and $X$ is its actual position in the needle reference coordinates as shown in Fig. 6 . The higher the stiffness the more important the constraint on the tool will be. However, the highest stable value of $k$ is mechanically limited by the haptic device and a low value of $k$ does not provide a good tool guidance. Hence, other types of guidance have been investigated. In a second approach, a guidance based on a sliding mode controller has been used. In [9], Jarillo-Silva proposed a model of the Geomagic Touch $^{\circledR}$ based on equation (2).

$$
M(\theta) \ddot{\theta}+V(\theta, \dot{\theta}) \dot{\theta}+G(\theta)=u
$$

where $M$ is the inertia matrix of the device, $V$ the centrifuge and Coriolis forces, $G$ the gravity forces, $u$ the torques applied to the motors and $\theta$ the angular position of the motors. From the equations presented in [10], $G$ is given by equation (3) where $k_{9}$ and $k_{10}$ are positive constants.

$$
G=\left[\begin{array}{c}
0 \\
k_{10} \times \cos \left(\theta_{2}\right)+k_{9} \times \sin \left(\theta_{3}\right) \\
k_{10} \times \cos \left(\theta_{2}\right)
\end{array}\right]
$$

In the model proposed by [10], the dissipation of the device is not taken into account, hence a friction coefficient has been added. With $u$ the torques applied by the motors to the joints and $\theta$ the vector of angular positions, the new dynamics are now:

$$
\ddot{\theta}=M^{-1} u-M^{-1}[G(\theta)+\{V(\theta, \dot{\theta})-b\} \dot{\theta}]
$$

In order to have a more concise model, let pose $g=M^{-1}$ and $f=M^{-1}[G(\theta)+\{V(\theta, \dot{\theta})-b\} \dot{\theta}]$. With $x_{1}=\theta$, $x_{2}=\dot{\theta}$ and taking into account the variable changes $\tilde{x}_{1}=$ $x_{1}-x_{1_{d}}$ and $\tilde{x}_{2}=x_{2}-x_{2_{d}}$, the new state model is given in eq. (5).

$$
\left\{\begin{array}{l}
\dot{\tilde{x}}_{1}=\tilde{x}_{2} \\
\dot{\tilde{x}}_{2}=g \cdot u-f-\dot{x}_{2_{d}}
\end{array}\right.
$$

Let $s$ be a sliding surface: $s=\lambda \tilde{x}_{1}+\tilde{x}_{2}$ where $\lambda$ is a vector with 3 positive values.

Let $V=\frac{s^{2}}{2}$ be a Lyapunov function. With $u=$ $[G(\theta)+\{V(\theta, \dot{\theta})-b\} \dot{\theta}]+M\left[\dot{x}_{2_{d}}-\lambda \tilde{\mathrm{x}}_{2}-C \cdot \operatorname{sgn}(s)\right]$, it can be shown that $V$ is globally positively defined and $\dot{V}$ is globally semi-defined negative. Hence, the system is stable. $C$ is a vector with three positive components and the sgn function is defined as follows:

$$
\begin{cases}\operatorname{sgn}(x)=1 & \text { if } \varepsilon<x \\ \operatorname{sgn}(x)=x / \varepsilon & \text { if }-\varepsilon \leq x \leq \varepsilon \\ \operatorname{sgn}(x)=-1 & \text { if } x<-\varepsilon\end{cases}
$$

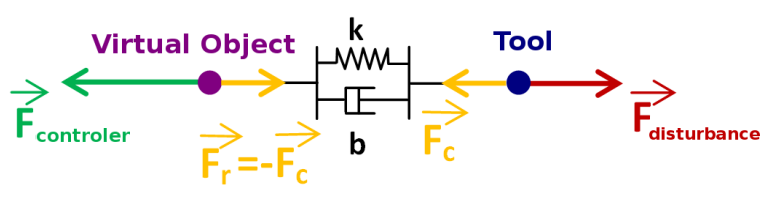

Fig. 7: Virtual fixture control

This control law is stable in Lyapunov's sense and provides the advantage to control independently the 3 motors and to optimize their own gains. However, the performances are not optimal. Indeed, putting high constraints at the frontier between two zones, induces a chattering phenomenon. When the gains are lowered to cancel this chattering, the guidance is not effective enough. Finally, a third method has been studied.

The virtual fixture control has been introduced by Colgate in [11]. In [12], Wilkening showed the interest of such a method for a guidance function. The principle is to apply a force to a virtual object linked by a spring-damper system to the tool, as illustrated in Fig. 7. The advantages of this system is to ensure the interaction stability at the cost of a limited perceived stiffness. This control law is adjustable using the stiffness $k$ of the spring, the damping coefficient $b$, the mass of the virtual object $M_{v o}$ and the force applied on the virtual object by the controller $F_{\text {controller. In addition, an }}$ higher $M_{v o}$ will stabilize the system even more. One of the drawbacks of this method is that the Mass-Spring-Damper system can oscillate at the frequency $\omega=\sqrt{\frac{K}{M_{o v}}-\frac{B^{2}}{2 m^{2}}}$. The parameters of the control law have been chosen so that the reasoning frequency is higher than $250 \mathrm{~Hz}$.

In [13], Kikuuwe proposed to compute $F_{\text {controller }}$ by using a sliding mode method. The main objective is to reproduce the characteristic of an anisotropic material which properties depend on the position and direction of displacement. The movements are penalized when the tool goes toward the forbidden zone and are facilitated when it tends to go back to the authorized zone. This control law has been implemented but, in our case, it did not lead effectively the tool back in the authorized region. In fact, the movement had to be initiated by the user.

Actually, $F_{\text {controller }}$ is computed using a ProportionalDerivative control law which provided experimental good results. The implemented control law is visible in equation (7) for the authorized zone and equation (8) for the forbidden zone. Table IV details the parameters used in the control law.

$$
\left\{\begin{aligned}
F_{\text {controller }} & =-b_{v o_{i n}} \cdot V_{v o} \\
F_{\text {tool }} & =K \cdot\left(X_{v o}-X_{o}\right)-b_{o_{i n}} \cdot V_{o} \\
M_{v o} \cdot \ddot{X}_{v o} & =F_{\text {tool }}-F_{\text {controller }}
\end{aligned}\right.
$$

$$
\left\{\begin{aligned}
F_{\text {controller }} & =k_{v_{o u t}} \cdot\left(X_{v_{o} d}-X_{v o}\right)-b_{v o_{o u t}} \cdot V_{v o} \\
F_{\text {tool }} & =K \cdot\left(X_{v o}-X_{o}\right)-b_{o_{\text {out }}} . V_{o} \\
M_{v o} \cdot \ddot{X}_{v o} & =F_{\text {tool }}-F_{\text {controller }}
\end{aligned}\right.
$$




\begin{tabular}{|c|c|}
\hline Parameters & Definition \\
\hline$X_{v o}[\mathrm{~m}]$ & Position of the virtual object \\
\hline$X_{O}[\mathrm{~m}]$ & Position of the tool \\
\hline$V_{v o}[\mathrm{~m} / \mathrm{s}]$ & Velocity of the virtual object \\
\hline$V_{o}[\mathrm{~m} / \mathrm{s}]$ & Velocity of the tool \\
\hline$K \quad[\mathrm{~N} / \mathrm{m}]$ & $\begin{array}{l}\text { Stiffness of the spring } \\
\text { between the tool and the virtual object }\end{array}$ \\
\hline$b_{o_{i n}}[\mathrm{Ns} / \mathrm{m}]$ & $\begin{array}{l}\text { Viscosity applied to the tool } \\
\text { when it is inside the authorized zone }\end{array}$ \\
\hline$b_{v o_{i n}}[\mathrm{Ns} / \mathrm{m}]$ & $\begin{array}{l}\text { Viscosity applied to the virtual object } \\
\text { when it is inside the authorized zone }\end{array}$ \\
\hline$k_{\text {voout }}[\mathrm{N} / \mathrm{m}]$ & $\begin{array}{l}\text { Stiffness applied on the virtual object } \\
\text { to bring it back to the authorized zone }\end{array}$ \\
\hline$M_{v o}[\mathrm{~kg}]$ & $\begin{array}{l}\text { Mass of the virtual object. It can be } \\
\text { different in the } 3 \text { directions }\end{array}$ \\
\hline$F_{\text {controller }}[\mathrm{N}]$ & $\begin{array}{l}\text { Force applied by the controller on the } \\
\text { virtual object }\end{array}$ \\
\hline$F_{\text {tool }}[\mathrm{N}]$ & Force applied by the device on the tool \\
\hline
\end{tabular}

TABLE IV: Parameters used in the virtual object control law

\section{B. Vertebrae contact}

In order to render the contact with a semi-hard surface, as described previously, the first attempt was to adapt the impulsion method introduced by Chang in [14] and by Constantinescu in [15]. The core idea is to apply an impulsion to the tool when it touches a virtual wall such that it is pushed back in the opposite direction. With $v_{0}$ and $v_{1}$ the velocities before and after the impact respectively, we want $v_{1}=-e \times v_{0}$. Noting $F_{p}$ the force applied by the device, $M$ its mass and $F_{h}$ the force applied by the operator, the dynamics of the haptic device before and after the impact are given by equation (9).

$$
\left\{\begin{array}{l}
M \dot{v}_{1}=F_{h}+F_{p} \\
M \dot{v}_{0}=F_{h}
\end{array}\right.
$$

Supposing the acceleration and the effort are constant during a period $T$, we have by integration over $T$ :

$$
\left\{\begin{array}{l}
M v_{1}=T F_{h}+T F_{p} \\
M v_{0}=T F_{h}
\end{array}\right.
$$

In the end, the force needed to be generated by the haptic device is:

$$
F_{p}=\frac{-(e+1) \cdot M \cdot v_{0}}{T}
$$

where $e$ is a positive constant. When $e=0$, the tool should stop. When $e=1$, the device should proceed in the opposite direction. Both cases were tried in our application but none of them gave a good rendering according to the anaesthesiologist involved in the project since the impulsions introduced more artefacts than improvements. The final decision was to simply implement a proportional controller depending on the depth of penetration. Since the Geomagic Touch ${ }^{\circledR}$ cannot generate a force high enough to simulate a hard contact, the rendering could not be perfect. Facing the limitations of this device, we plan to implement this system on a more powerful one.

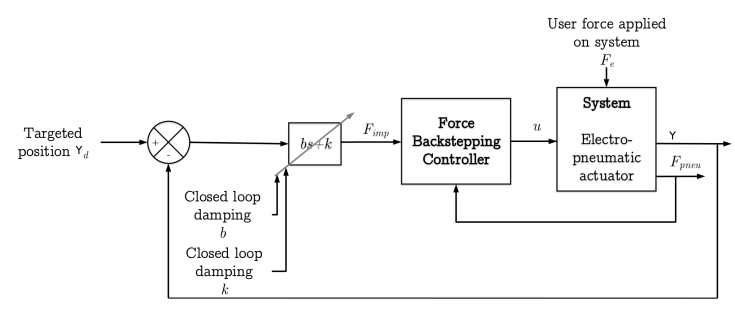

Fig. 8: Impedance controller block diagram

\section{Pneumatic actuator and loss of resistance}

As explained in section II, the electropneumatic actuator should display a stiffness between $100 \mathrm{~N} / \mathrm{m}$ and $3000 \mathrm{~N} / \mathrm{m}$. It should also be controlled in position in order to reproduce the liquid leakage in the patient's back. The characteristics of the electropneumatic components used to implement the model are given in table V.

\begin{tabular}{|c|c|}
\hline Component & Reference \\
\hline Cylinder & Airpel M16DD100D \\
\hline Servovalve & Festo MYPE-5-010-B \\
\hline Position sensor & MEAS-SPEC 2000 DC-EC LVDT \\
\hline Pressure sensor & MEAS-SPEC U5100 \\
\hline Controller board & dSPACE DS1104 \\
\hline
\end{tabular}

TABLE V: Electropneumatic components used on the test bench

To control the electropneumatic actuator in position with a variable stiffness, an impedance controller has been designed. This controller is composed of two nested loops. The inner loop is a force controller obtained by backstepping synthesis from an electropneumatic model described in [16] or [17]. The outer loop is a Proportional Derivative (PD) controller in position. Thus, the PD gain can be seen as the stiffness and the damping of the electropneumatic actuator. These gains can be tuned in real time, so the stiffness of the electropneumatic actuator can be controlled to simulate the syringe's stiffness variation during the epidural needle insertion simulation. The block diagram of the impedance controller is given Fig. 8. Where $Y_{d}$ and $Y$ are respectively the desired and the measured piston position, $F_{i m p}$ is the reference force for the force backstepping controller, $u$ is the reference voltage applied to the servovalve, $F_{\text {рпеи }}$ is the pneumatic actuator force, $b$ and $k$ are respectively the desired stiffness and damping of the electropneumatic actuator, and $F_{e}$ is the force applied by the user on the electropneumatic actuator. It has to be noticed that $F_{e}$ is not measured and it is seen as a disturbance. $Y_{d}$ is set dynamically in order to represent the fluid leakage into the patient's back.

Two experimental tests have been realized on this controller. The first one is a sinusoidal position tracking and the second one quantifies the static stiffness error for different perturbations. For the sinusoidal position tracking, the desired trajectory is given by: $Y_{d}=0.02 \cos (2 \pi t)$. The stiffness and damping are respectively set to $k=3000 \mathrm{~N} / \mathrm{m}$ and $b=200$ N.s/m. Fig. 9 displays the response of the electropneumatic actuator and shows that the trajectory is globally followed. 


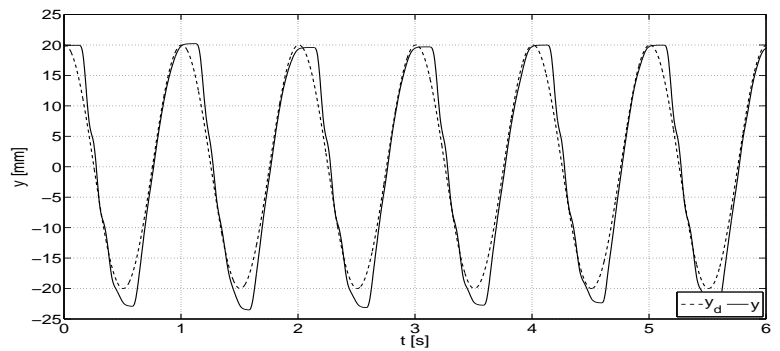

Fig. 9: 1Hz Sinusoidal tracking position

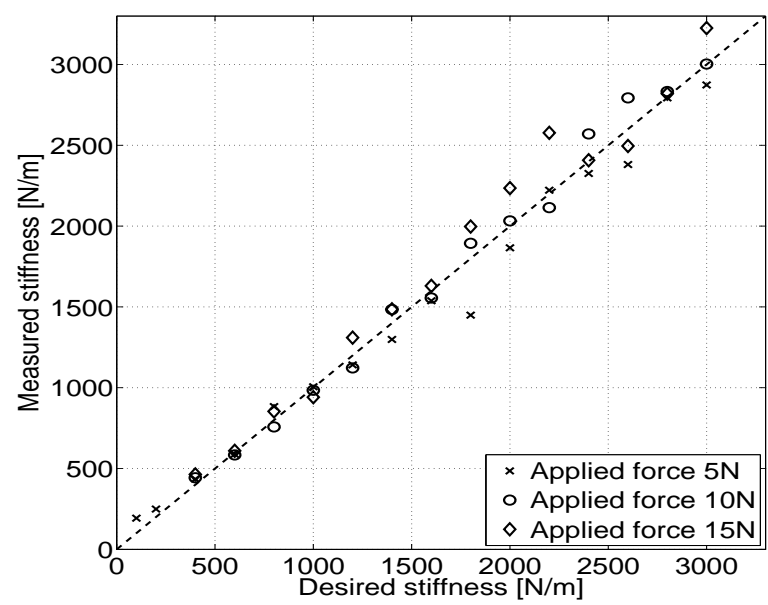

Fig. 10: Impedance controller stiffness static error

The maximal tracking error is about $7 \mathrm{~mm}$ over a range of $40 \mathrm{~mm}$.

Finally, the second experiment checks that the stiffness can be set to different values between $100 \mathrm{~N} / \mathrm{m}$ and $3000 \mathrm{~N} / \mathrm{m}$. This time, the position reference is set to $Y_{d}=0$, and three different perturbations $F_{e}$ are applied: $5 \mathrm{~N}, 10 \mathrm{~N}$, and $15 \mathrm{~N}$. This protocol has been repeated for various stiffness values of $k \in[100 ; 3000]$. The results are given on Fig. 10. These results show that the stiffness control is globally reached, but the error raises with the disturbance force and the desired stiffness.

\section{CONCLUSIONS}

In this paper, we presented the different control laws and models we experimentally tried in order to design a novel epidural needle insertion simulator. The final different control laws, linked with a new 3D patient parametric model and applied on an electrical haptic device (Geomagic Touch ${ }^{\circledR}$ ) combined with an electropneumatic actuator, render the needle and the L.O.R. syringe behaviors during the insertion into the biological layers. The main limitation encountered during the experimental trials is linked to the electric haptic device which is not sufficiently powerful to deliver the necessary levels of forces needed for the rendering. Nevertheless, this study showed that it was possible to develop a new epidural insertion simulator by using an electrical device to render the force on the needle and a pneumatic actuator to render the stiffness and fluid leakage of the syringe. The next task will consist in implementing this model on an interface that can render higher forces. Final user trials will then be conducted to validate the effectiveness of the simulator for this kind of training.

\section{ACKNOWLEDGMENT}

The authors would like to thank the ANR (French National Research Agency) for financing SAMSEI project (ANR-11IDFI-0034) under the supervision of Pr. X. Martin.

\section{REFERENCES}

[1] N. Vaughan, V. N. Dubey, M. Y. Wee, and R. Isaacs, "A review of epidural simulators: Where are we today?" Medical Engineering \& Physics 35, pp. 1235-1250, 2013.

[2] V. Manoharan, "Epidural needle insertion simulator. a device for training resident anaesthesiologists," Master's thesis, Delft University of Technology, 2011.

[3] D. Tran, K.-W. Hor, A. A. Kamani, V. A. Lessoway, and R. N. Rholing, "Intrumentation of the loss-of-resistance technique for epidural needle insertion," IEEE Transactions on Biomedical Engineering, vol. 56, pp. 820-827, March 2009.

[4] C. M. Gleeson and F. Reynolds, "Accidental dural puncture rates in uk obstetric practice," International Journal of Obstetric Anesthesia, vol. 7, pp. 242-246, 1998.

[5] A. Ghaleb, "Postdural puncture headache," Anesthesiology Research and Practice, vol. 2010, 2010.

[6] V. Manoharan, D. van Gerwen, J. J. van den Dobbelsteen, and J. Dankelman, "Design and validation of an epidural needle insertion simulator with haptic feedback for training resifent anaesthesiologist," in Proc. of the IEEE Haptics Symposium, Vancouver, BC, Canada, March 2012, pp. 341-348.

[7] P. N. Brett, T. J. Parker, T. A. Thomas, and A. Carr, "Simulation of resistance forces acting on surgical needles," The Institution of Mechanical Engineers IMechE, vol. 211, no. H, pp. 335-346, 1997.

[8] T. Dang, T. M. Annaswamy, and M. A. Srinivasam, "Development and evaluation of an epidural injection simulator with force feedback for medical training," Stud Health Technol Inform, vol. 81, pp. 97-102, 2001.

[9] A. Jarillo-Silva, O. A. Dominguez-Ramirez, V. Parra-Vega, and J. P. Ordaz-Oliver, "Phantom omni haptic device: Kinematic and manipulability," in Proc. of the IEEE Electronics, Robotics and Automotive Mechanics Conference, Cuernavaca, Morelos, Mexico, Sept. 2009, pp. 193-198.

[10] T. Sansanayuth, I. Nilkhamhang, and K. Tungpimolrat, "Teleoperation with inverse dynamics control for phantom omni haptic device," in SICE Annual Conference, Akita, Japan, Aug. 2012, pp. 2121-2126.

[11] J. E. Colgate, M. C. Stanley, and J. M. Brown, "Issues in the haptic display of tool use," in Intelligent Robots and Systems 95. 'Human Robot Interaction and Cooperative Robots', Proceedings. 1995 IEEE/RSJ International Conference on, vol. 3, Aug 1995, pp. 140-145 vol.3.

[12] P. Wilkening, W. Chien, B. Gonenc, J. Niparko, J. U. Kang, I. Iordachita, and R. H. Taylor, "Evaluation of virtual fixtures for robotassisted cochlear implant insertion," in IEEE RAS \& EMBS International Conference on Biomedical Robotics and Biomechatronics (BioRob), Sao Paulo, Brazil, Aug. 2014.

[13] R. Kikuuwe, N. Takesue, and H. Fujimoto, "Passive virtual fixtures based on simulated position-dependent anisotropic plasticity," in Proc. of the IEEE International Conference on Robotics and Automation (ICRA 2007), 2007, pp. 3263-3268.

[14] B. Chang and J. E. Colgate, "Real-time impulse-based simulation of rigid body systems for haptic display," in Proc. of the ASME Interational Mechanical Engineering Congress and Exhibition, 1997.

[15] D. Constantinescu, S. E. Salcudean, and E. A. Croft, "Haptic rendering of rigid contacts using impulsive and penalty forces," IEEE Transactions on Robotics, vol. 21, no. 33, pp. 309-323, June 2005.

[16] B. Taheri, D. Case, and E. Richer, "Force and stiffness backsteppingsliding mode controller for pneumatic cylinders," IEEE ASM Transactions on mechatronics, vol. 19, no. 6, 2014.

[17] F. Abry, X. Brun, S. Sesmat, E. Bideaux, and C. Ducat, "Electropneumatic cylinder backstepping position controller design with realtime closed-loop stiffness and damping tuning," IEEE Transactions on Control Systems Technology, vol. PP, no. 99, 2015. 